\title{
Neutron spectrometry at JET (invited)
}

Cite as: Review of Scientific Instruments 57, 1717 (1986); https://doi.org/10.1063/1.1138559

Published Online: 04 June 1998

O. N. Jarvis, G. Gorini, M. Hone, J. Källne, G. Sadler, V. Merlo, and P. van Belle

\section{(1)}

\section{ARTICLES YOU MAY BE INTERESTED IN}

A diamond based neutron spectrometer for diagnostics of deuterium-tritium fusion plasmas Review of Scientific Instruments 85, 11 E101 (2014); https://doi.org/10.1063/1.4885356

Compact NE213 neutron spectrometer with high energy resolution for fusion applications Review of Scientific Instruments 75, 3553 (2004); https://doi.org/10.1063/1.1787935

Silicon surface barrier detector for fusion neutron spectroscopy

Review of Scientific Instruments 57, 1763 (1986); https://doi.org/10.1063/1.1139174

\section{Challenge us.}

What are your needs for periodic signal detection?

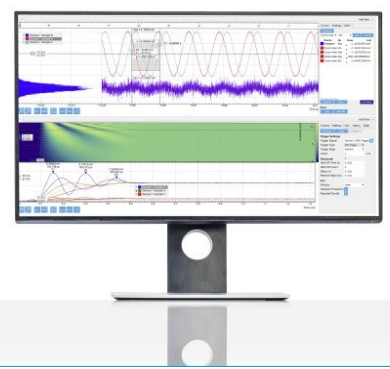

Zurich Instruments 


\title{
Neutron spectrometry at JET (invited)
}

\author{
O. N. Jarvis, G. Gorini, M. Hone, J. Källne, G. Sadler, V. Merio, and P. van Belle \\ JET Joint Undertaking, Abingdon, Oxon, OX14 3EA, United Kingdom
}

(Presented on 10 March 1986)

\begin{abstract}
Measurements of the energy spectra of fusion neutrons is accorded a prominent position in the neutron diagnostics program at JET. The nature of the information on plasma properties which it is hoped will be obtained is discussed, as is the availability of suitable instruments and techniques for spectrometry on thermonuclear plasmas. Of these instruments, only the ${ }^{3} \mathrm{He}$ ionization chamber has, so far, been successfully exploited; the lessons learned regarding its performance are outlined and the results obtained for ohmically and ICRF-heated plasmas are presented. It is shown that the deuterium ion energy distributions appear to be Maxwellian for all plasmas studied, that central ion temperatures have been obtained on a shot-by-shot basis which agree well with corresponding values obtained by neutral particle analysis and that the deuteron-to-electron density ratio in the plasma is approximately 0.5 .
\end{abstract}

\section{NTRODUCTION}

Neutron spectrometry has long had a part to play in fusion research. ${ }^{1}$ The original motivation was to try to confirm the thermonuclear origin of the neutron emission from the various fusion devices but, eventually, the merits of high resolution neutron spectrometry were appreciated ${ }^{2,3}$ as an ion temperature diagnostic. In recent years a few attempts to realize this potential have been made $e^{4-6}$ and these can be considered to have provided proof of principle. However, it is only with the advent of JET and TFTR with their high neutron yields and long pulse durations that neutron spectrometry is expected to become a routine diagnostic.

That the exploitation of neutron spectrometry as a plasma diagnostic is still at such a tentative stage is due to two factors. First, there is no spectrometer available which provides the detection efficiency and energy resolution needed for operational use with the low neutron emission intensities from the smaller magnetic confinement devices. Second, the neutral particle charge exchange technique ${ }^{7}$ has been found to provide reliable data on hydrogenic ion energy distributions with useful spatial and time resolution. Because charge-exchange diagnostics encounter increasing difficulties as the plasma size, density, and neutron production increase, it is presently considered that they will not be operable during high neutron yield D-T plasma operation, for which neutron spectrometry should at last assume a role of major importance.

\section{OBJECTIVES}

The primary objective for neutron spectrometry is the identification of the production mechanisms responsible for the observed neutron emission. It is clearly indefensible to embark on a program of research and development into a fusion power source which depends on the production of neutrons as an essential component of the energy cycle without confirming that the relevant physics is fully understood. This basic understanding should be established during deuterium plasma operation. It is sometimes argued, however, that by the time D-T plasma operation commences the neutron production mechanisms will be sufficiently well understood that the only neutron measuring instrument really needed will be a simple neutron counter. This view would perhaps be correct if plasma ignition could be realized by the brute force addition of heating power. Unfortunately, recent experience ${ }^{8}$ has shown that a successful outcome will not be obtained so easily with the present generation of tokamaks. Nevertheless, significant $\alpha$-particle heating is expected and the study of $\alpha$-particle confinement can be commenced. It is in just this situation that neutron spectrometry can be particularly helpful.

The identification of neutron production mechanisms is a broad field of endeavor which is conveniently divided into two, depending on whether coarse or fine neutron energy resolving power is required of the spectrometer employed. Coarse energy resolution should suffice to distinguish electron processes (electrodisintegration of deuterium fuel and photoneutron production at limiters) from fusion processes, to separate $\mathrm{D}-\mathrm{D}$ from $\mathrm{D}-\mathrm{T}$ and $\mathrm{T}-\mathrm{T}$ fusion reactions and to identify the secondary $T-D$ neutrons ${ }^{9}$ emitted from deuterium plasmas, whereas very good energy resolution is needed to distinguish fusion reactions resulting from thermal effects from those due to the presence of fast particles introduced by the various additional heating techniques. These various contributions can be identified by the terminology "plasmaplasma," "beam-plasma," and "beam-beam" induced fusion reactions, where the term "beam" is applied here equally to those beam ions injected as neutral particle heating beams as to those high-energy (or, at least, nonthermal) particles generated through ICRF heating. Good resolution is also required to measure the energy spectrum of the secondary T-D neutrons emitted from deuterium plasmas; this measurement would test the presence of nonclassical fast particle loss mechanisms.

An investigation into production mechanisms might appear to be unconnected with the study of high-temperature plasmas but the results of the investigation are expressible in more familiar terms. The measured neutron energy spectra are directly related to the energy distribution in the plasma 


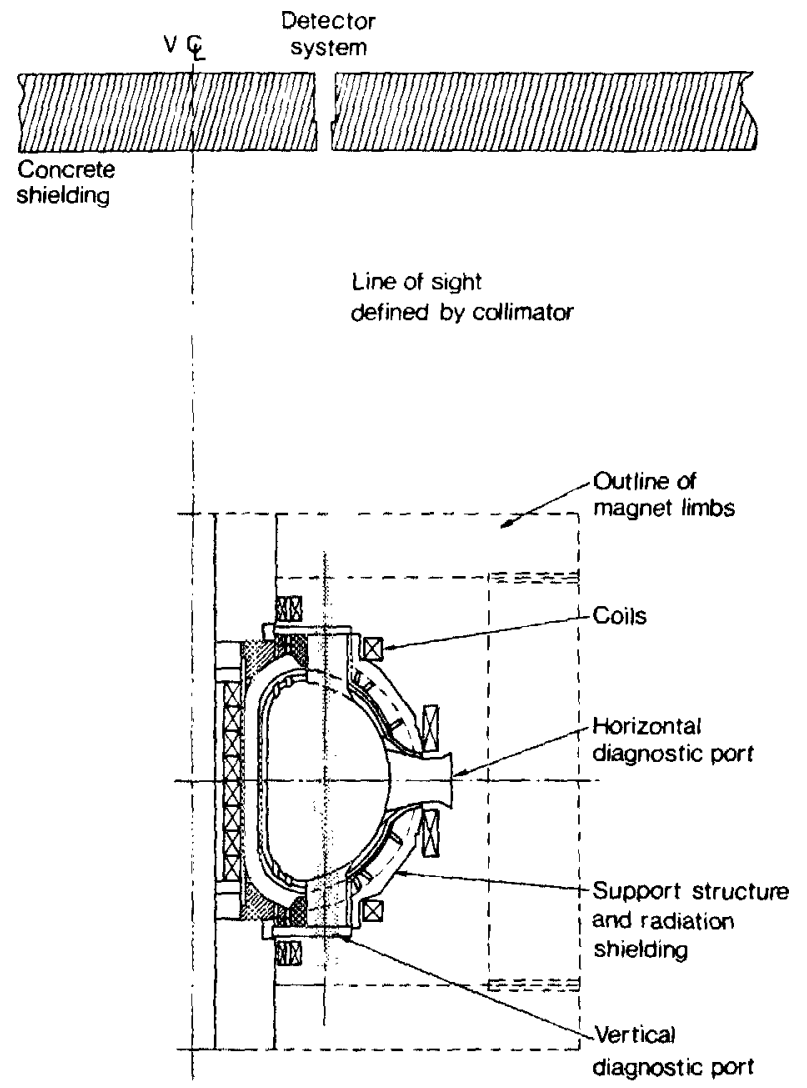

Frg. 1. Schematic layout of the line of sight to the Roof Laboratory.

of the reacting ions which, in the case of additionally heated plasmas, is dependent on a whole range of plasma and heating technique properties. The extent to which the many physics parameters can be disentangled depends on circumstances. However, for plasma species in thermal equilibrium, the central ion temperature is readily extracted along with valuable information concerning the hydrogen ion densities, which are not directly measured by conventional diagnostic techniques.

\section{INSTRUMENTS AND METHODS}

An essential prerequisite for successful measurements of neutron energy spectra is an operating position for the spectrometer which permits the plasma to be viewed along a well-collimated line of sight but which is otherwise heavily screened from neutron and gamma radiation emanating from the fusion device. Provided the neutron yield is sufficiently high, the ideal viewing location is outside the biological shield wall. At JET we have two such locations in the Roof Laboratory, with the collimated neutron beams being permitted to escape from the building in the vertical direction (Fig. I). In order to obtain a line of sight tangential to the plasma current, a movable containment for the spectrometer will be placed within the Torus Hall itself (Fig.2).

With spectra taken along a single line of sight it will probably prove difficult to separate beam-beam, beam-plasma, and plasma-plasma contributions, but with two comple-

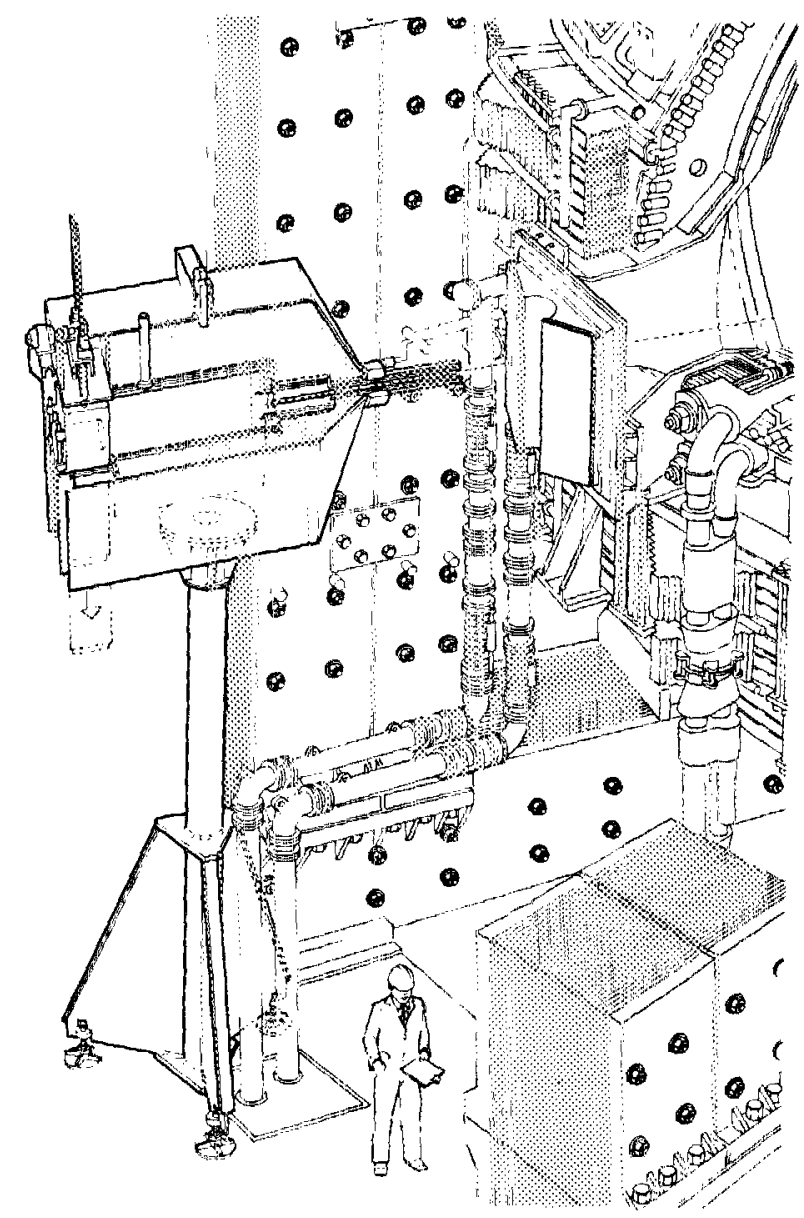

FIG. 2. Layout of the neutron spectrometer radiation shield in the Torus Hall.

mentary lines of sight which emphasize these contributions differently, we can hope to obtain a more detailed understanding by exploiting the kinematic properties of the twoparticle reactions ${ }^{10}$ coupled, for deuterium plasmas, with the effects of the nonisotropic reaction cross section. The kinematic dependence is easy to appreciate in the case of a cylindrical plasma, with neutral beams injected axially; in this case the axial, but not the radial, line of sight would provide spectra Doppler shifted to higher or lower energies by the forward motion of the beam ions and also by any axial bulk motion of the plasma column. In JET, these differences are expected to be smeared out to some extent because our "tangential" line of sight is really tangential to the inner wall of the vacuum vessel rather than to the axis of the plasma discharge and also because the neutral beams are injected over a wide range of angles to the field lines rather than axially. Nevertheless, the vertical line of sight will be sensitive mainly to the normal component of velocity of the beam ions whereas the tangential line of sight will be sensitive mainly to the parallel component. Fast ions generated by ICRF heating will possess velocities primarily normal to the magnetic field lines.

Several types of $2.5-\mathrm{MeV}$ neutron spectrometers are currently being prepared for use with deuterium plasmas, as listed in Table $\mathrm{I}$. 
TABLE I. Spectrometer for $2.5-\mathrm{MeV}$ neutrons

\begin{tabular}{lcc}
\hline $\begin{array}{l}\text { Spectrometer type } \\
\text { for D-D plasmas }\end{array}$ & $\begin{array}{c}\text { Energy } \\
\text { resolution (\%) }\end{array}$ & $\begin{array}{c}\text { Efficiency } \\
\text { (counts/n cm }{ }^{-2} \text { ) }\end{array}$ \\
\hline NE213 scintillator & 8 & 1 \\
Nuclear emulsions & 4 & $10^{-3}$ \\
$\mathrm{H}_{2}$ spherical ion chamber & 3 & $10^{-2}$ \\
${ }^{3}$ He ion chamber & 1.5 & $10^{-2}$ \\
Neutron time of flight & 4 & $10^{-3}$ \\
Proton recoil (thin foil) & 7 & $10^{-6}$ \\
\hline
\end{tabular}

The required energy resolution $(R)$ and efficiency $(\eta)$ can be specified by demanding the spectrometer to provide useful results for ohmically heated plasmas at an operating position $20 \mathrm{~m}$ distant: our ideal spectrometer would then have $R \sim 4 \%, \eta>10^{-2}$ counts $/ \mathrm{n} \mathrm{cm}^{-2}$ and would possess a wide dynamic range of operation. The spectrometers listed in Table I all possess drawbacks, as discussed previously. ${ }^{\text {" }}$ The recently installed neutron time-of-flight spectrometer ${ }^{12}$ has been designed specifically to cover a wide dynamic range but, due to its inherent background radiation sensitivity, it is suitable only for deployment in the Roof Laboratory. The ${ }^{3} \mathrm{He}$ ion chamber offers excellent energy resolution and efficiency, but suffers from certain problems which are exposed later in this paper. A particular concern regarding its use in the Torus Hall is its high sensitivity to epithermal neutrons. It is possible that the hydrogen spherical ionization chamber $^{13}$ will give better results, at least until fluxes are high enough for the proton-recoil technique to become useful. Nuclear emulsions ${ }^{14}$ will be employed for confirmatory purposes. The chief role of the NE213 scintillator is as a moderate resolution $2.5-\mathrm{MeV}$ neutron flux monitor.

Table II identifies the spectrometer types under serious consideration for use with D-T plasmas.

Ideally, the required spectrometer energy resolution and efficiency as determined for D-T plasmas with additional heating applied are $R \sim 2 \%$ and $\eta>3 \times 10^{-5}$ counts $/ \mathrm{n} \mathrm{cm}^{-2}$. The Si diode spectrometer ${ }^{11}$ is expected to be usable with the narrow energy spectra provided by ohmically heated base plasmas. The tandem-radiator approach, ${ }^{15}$ a thin-foil proton-recoil technique, is suitable for operation within a movable containment in the Torus hall; the two time-of-flight spectrometers ${ }^{16,17}$ are not.

\section{INI. PREDICTHON OF NEUTRON ENERGY SPECTRA}

A supporting activity of considerable importance is the development of a computer code which can predict the neutron energy spectra which should be recorded along any line

TABLE II. Spectrometer for 14-MeV neutrons.

\begin{tabular}{lcc}
\hline $\begin{array}{l}\text { Spectrometer type } \\
\text { for D-T plasmas }\end{array}$ & $\begin{array}{c}\text { Energy } \\
\text { resolution }(\%)\end{array}$ & $\begin{array}{c}\text { Efficiency } \\
\left(\text { counts } / \mathrm{n} \mathrm{cm}^{-2}\right)\end{array}$ \\
\hline Nuclear emulsions & 3 & $10^{-4}$ \\
Si $\left(n, \alpha_{0}\right)$ reaction & 0.7 & $10^{-3}$ \\
Tandem radiator method & 2.4 & $10^{-4}$ \\
Associated-particle time of flight & 1.4 & $10^{-5}$ \\
$\mathrm{D}_{2}$ backscatter time of flight & 1.0 & $10^{-3}$ \\
\hline
\end{tabular}

of sight for given conditions of plasma heating (e.g., number and orientation of injected neutral beams, etc.) and for set values of such plasma parameters as temperature and density profiles. The computer code under development ${ }^{18}$ for JET is called NSPEC. Important features of the code are power deposition, a solution to the Fokker-Planck equation for the fast ion velocity distribution, and the use of relativistic kinematics including anisotropic reaction cross sections.

Previous calculations of neutron spectra from beamheated plasmas have been restricted ${ }^{19}$ to simultaneous equal intensity co- and counter-injection of monoenergetic beams in the parallel direction and parallel observation. This idealized geometry clearly demonstrated the importance of $Z_{\text {eff }}$ in determining the fast ion velocity distribution through pitch-angle scattering.

Given both experimental results and reliable predictions, the next step will be to perform a multiparameter search to optimize the fit of prediction to data. This step has not yet been taken, although neutron spectra from beamheated plasmas have been measured. ${ }^{4,14}$

\section{MEASUREMENTS}

Neutron spectrometry measurements on JET plasmas have, so far, been concentrated on the exploitation of the ${ }^{3} \mathrm{He}$ ionization chamber. Detectors of this type have previously been used in tokamak research, first by Strachan ${ }^{4}$ on PLT and, more recently, by Fisher ${ }^{5}$ on Alcator C. In both instances the neutron fluxes were low and only very limited statistics were acquired.

The particular ${ }^{3} \mathrm{He}$ ionization chamber used on JET was a commercial item, marketed by Seforad ${ }^{20}$ It is a cylindrical, gridded, chamber filled with ${ }^{3} \mathrm{He}, \mathrm{Ar}$, and $\mathrm{CH}_{4}$ to a total pressure of about $10 \mathrm{~atm}$. It was placed in the Roof Laboratory, with its axis horizontal, such that its active volume was fully immersed in the neutron flux originating from the plasma some $20 \mathrm{~m}$ below. About $4 \%$ of the fusion neutrons passing through the chamber undergo ${ }^{3} \mathrm{He}(n, p) t$ reactions $(Q$ value $763.8 \mathrm{keV}$ ); the two fast particles transfer their entire energy to the gas through excitation and ionization processes provided they do not encounter the chamber walls before coming to rest. The charge collected by the central wire is thus proportional to the total energy released in the reaction $\left(Q+E_{n}\right)$. The pulse-height spectrum therefore comprises a full-energy peak for the $28 \%$ of the events for which the two charged particles do not encounter the walls, and a broad low-energy tail for the $72 \%$ which do. In addition, the detector is very sensitive to thermal and epithermal neutrons owing to the $1 / v$ dependence of the $n\left({ }^{3} \mathrm{He}, t\right) p$ reaction cross section. As a result, we find that the ratio of full energy to total events is about 1:50.

An essential preliminary to the use of the ion chamber on JET was the measurement of its energy-dependent response function. In the present work, the source of monokinetic neutrons was the $T(p, n)^{3} \mathrm{He}$ reaction using protons with energies up to $3.20 \mathrm{MeV}$ from a 3-MV Van de Graaff incident on a $50 \mu \mathrm{g} / \mathrm{cm}^{2}$ thick $\mathrm{Ti} / T$ target viewed at $0^{\circ}$ relative to the beam direction. The overall energy spread in the resulting neutron beam was less than $15 \mathrm{keV}$. The detector 


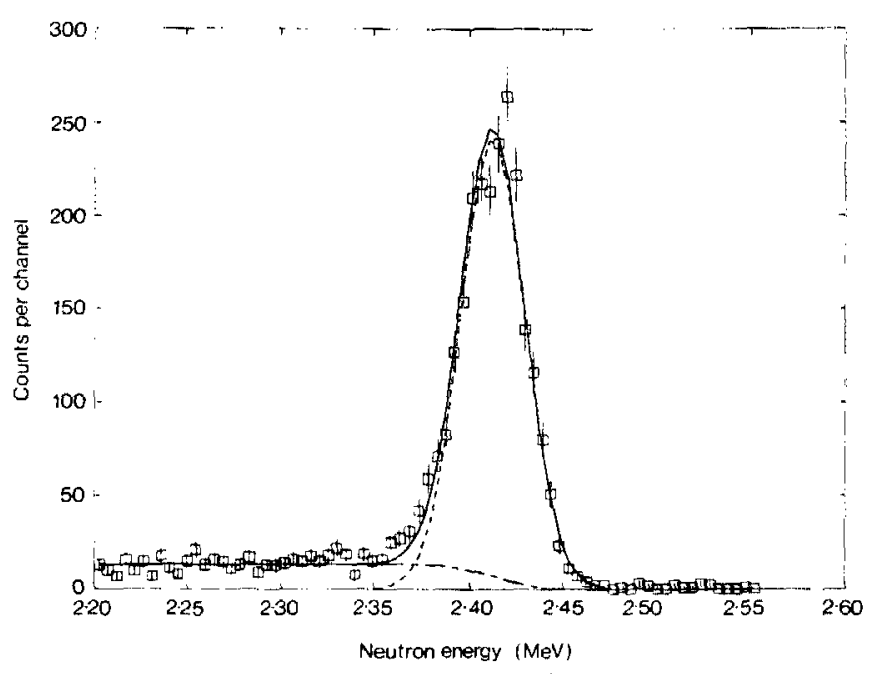

FIG. 3. Showing the response of the ${ }^{3} \mathrm{He}$ ion chamber to $2.42-\mathrm{MeV}$ neutrons.

response to 2.42-MeV neutrons is shown in Fig. 3, where the full energy peak is seen to be nearly Gaussian in form with FWHM of $44 \mathrm{keV}$. While the response function does vary with energy, this variation is slight over the range of interest in analyzing ohmic heated spectra and was neglected in the present work.

A typical pulse-height spectrum obtained at JET with an ohmically heated plasma is shown in Fig. 4. The method of analysis adopted was to assume a Gaussian form of unknown width $W$ and height $H$ for the fusion neutron spectrum, to convolute this with the response function and to fit the result to the measured pulse-height spectrum using a maximum-likelihood ${ }^{21}$ method to obtain optimum values for $W$ and $H$. This procedure is applicable only to neutron emission from plasmas in thermal equilibrium, for which the emitted neutron spectra are ${ }^{3}$ nearly Gaussian in form, with $\mathrm{FWHM}=82.5 \sqrt{T_{i}}$ for deuterium plasmas at temperature $T_{i}(\mathrm{keV})$.

One of the major limitations with the ${ }^{3} \mathrm{He}$ ionization chamber is its restricted count-rate capability (a few $\mathrm{kHz}$ only) which results from the need to use long time constants $(10 \mu \mathrm{s})$ in order to optimize the energy resolution. A simple analysis ${ }^{11}$ shows that when both response function and neutron spectrum under investigation can be described by Gaussian functions with FWHM values $R$ and $W$, respectively, then the relation between $\Delta T_{i} / T$ and total count $N$ in the measured pulse-height spectrum is $\Delta T_{i} / T_{i}$ $=\left(1+R^{2} / W^{2}\right) 2^{1 / 2} N^{-1 / 2}$. For $R \ll W$, a $10 \%$ temperature measurement requires but 200 counts in the spectrum.

Unfortunately, our response function is not a simple Gaussian and the amplitude of the low-energy tail due to the wall effect contributes in a major way to the "effective" resolution obtained by forming the second moment of the response function over the width of the neutron spectrum. This effect has been studied numerically ${ }^{22}$ over the range 1$10 \mathrm{keV}$ with the result $\Delta T_{i} / T_{i}=\alpha 2^{1 / 2} N^{-1 / 2}$, with $\alpha=1.81 \pm 0.01$, instead of the expected $\alpha=1.08$ for a pure $42 \mathrm{keV}$ wide Gaussian.

In other words, we obtain $R \approx W$ under all circumstances and the excellent energy resolution apparently of-

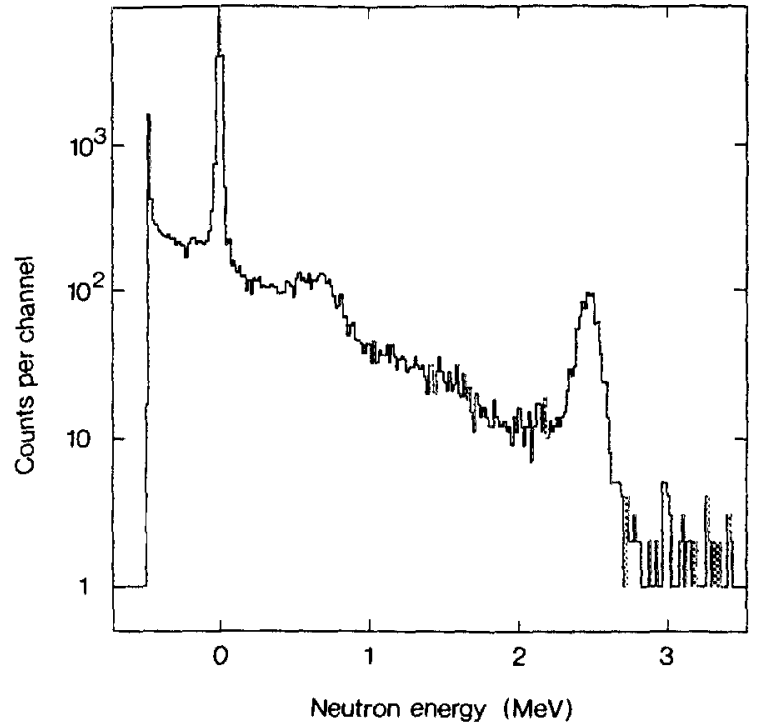

FIG. 4. A pulse-height spectrum obtained from an ohmically heated plasma.

fered by the ${ }^{3} \mathrm{He}$ ionization chamber proves to be illusory. A good spectrum requires not 200 counts, but 660 . With a maximum acceptable count rate of $5 \mathrm{kHz}$, the minimum measuring period for a $10 \%$ accurate ion temperature value is $6.6 \mathrm{~s}$.

There is one further detail to be discussed. So far we have been considering neutron emission as being from a plas$\mathrm{ma}$ at $\mathrm{a}$ uniform density and temperature. In practice, the neutron emission is a line integral over a plasma for which the precise ion density and temperature profiles are unknown but the emission is strongly weighted to the central dense and hot core of the plasma. A numerical study using sensible profiles indicates that the Gaussian spectrum shape is realistic but that the central ion temperature can be obtained from the apparent temperature by multiplying with the factor $1.08 \pm 0.01$. The effect of sawtooth oscillations on the time-averaged central temperature is believed to be negligible.

Our spectrometry measurements on JET have been restricted to 36 plasma discharges which provided a sufficiently high neutron flux and discharge duration that time-averaged temperatures could be obtained to at least $\pm 20 \%$ on a single discharge basis. These were all ohmically heated discharges with $B_{T}=3.4 \mathrm{~T}, I_{p}$ in the range 3-5 MA, central. electron density $2.4-5.5 \times 10^{13} \mathrm{~cm}^{-3}$ and central electron temperatures $2.8-4.0 \mathrm{keV}$. A group of pulse-height spectra taken for consecutive discharges is illustrated in Fig. 5. Since the discharges in this cluster were nearly identical, the data have been aggregated and the fit to this data set is displayed in Fig. 6. That this fit is statistically acceptable indicates that our underlying assumptions are reasonable. In particular, the spectrum lacks any vestige of the high-energy tail that would accompany a high-energy component in the ion-energy distribution and is apparently free from low-energy neutrons resulting from scattering of the thermonuclear neutrons from the vacuum vessel walls and windows.

The aggregate spectrum provides a sufficiently precise value for $T_{i}$ that this result can be combined ${ }^{6}$ with the total 


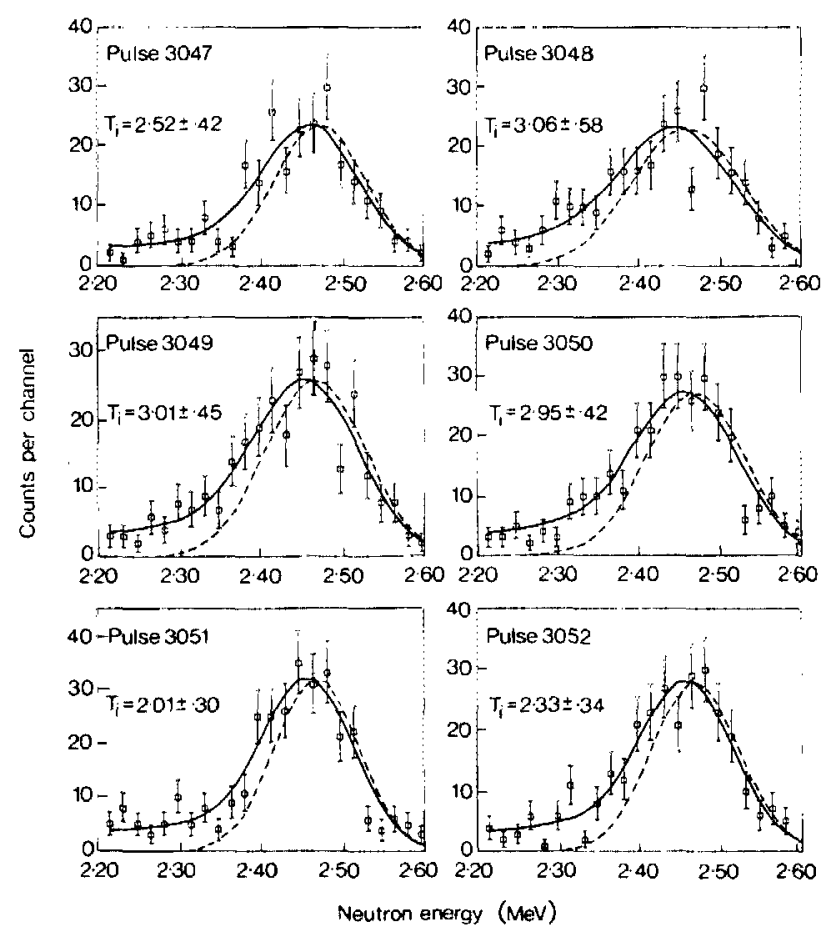

FIG. 5. High-energy portions of pulse-height spectra for six consecutive discharges, showing deduced Gaussian neutron spectra (dotted) and fitted response curves (continuous).

neutron yield, which scales as $n_{d}^{2} T_{i}^{\alpha}$ where $\alpha \simeq 4$ for $T_{i}=3$ $\mathrm{keV}$ to provide a measure of $n_{d}$ (or, rather $n_{d} / n_{e}$ ). In this way, we find $n_{d} / n_{e}=0.47 \pm 0.07$ for the spectra illustrated in Figs. 5 and 6. Other aggregated spectra provide confirmatory density ratios. It should be noted that such a low-density ratio is rather difficult to reconcile with the optical spectroscopy and bremsstrahlung data; a ratio of $0.6-0.7$ is indicated by these diagnostics. ${ }^{23}$

An idea of the $T_{i}$ time dependence can be obtained by aggregating spectra for corresponding 1-s time intervals. One such set of measurements is displayed in Fig. 7, where it is seen that the spectrometer temperatures follow closely the neutron yield temperatures (now assuming $n_{d}=0.5 n_{\varepsilon}$ ).

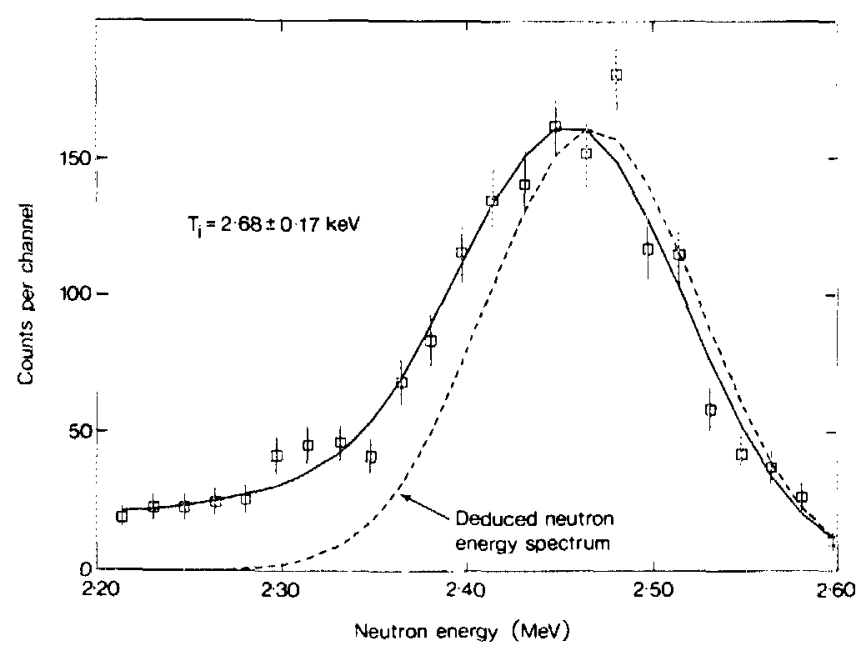

Fig. 6. The pulse-height spectrum obtained by summing over discharges 3047 to 3052 .

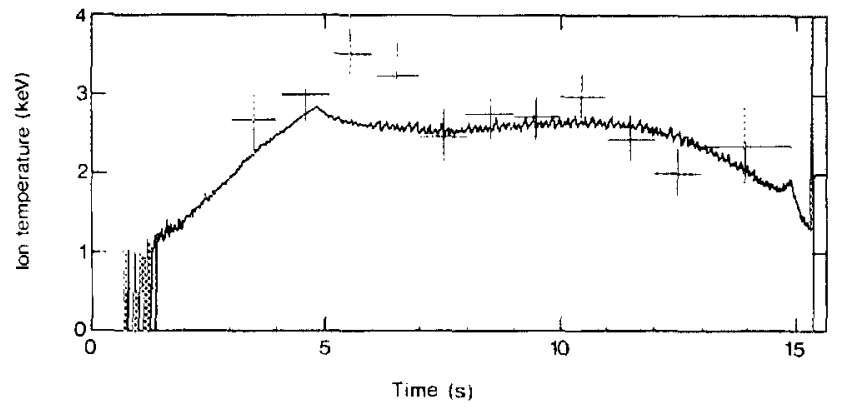

FIG. 7. Time dependence of the central ion temperature measured by neutron spectrometry, summing over 12 similar discharges, superimposed on the temperature trace obtained from the neutron yield measurement for discharge 3052 .

The ion temperature data obtained from the neutron spectrometer agree well with the neutral particle analyzer measurements. ${ }^{24}$ Averaging the data for all 36 discharges gives a ratio of neutron ion temperature to NPA temperature of $1.07 \pm 0.013$ (excluding any NPA systematic errors).

Finally, some 23 ICRF-heated plasmas with ${ }^{3} \mathrm{He}$ as minority species were also studied. The discharges were all at 4.0 MA and 3.4 $\mathrm{T}$ but the period during which the rf power was held constant was only $1 \mathrm{~s}$ in duration, so these discharges had to be aggregated before analysis. The increase in ion temperature was of order $10 \%$ for 2-MW applied rf power. Most notably, the neutron energy spectra taken during ICRF heating showed no sign of the high-energy tail which would indicate a nonthermal component in the deuterium ion energy distribution. This lack of nonthermal deuterium ions was confirmed by the NPA measurements.

\section{CONCLUSIONS}

The importance of neutron spectrometry for high-temperature plasma diagnosis in fusion devices operating with deuterium and deuterium-tritium plasmas has been outlined. The instruments and techniques available for making the measurements have been described and the need for further work indicated. Finally, the measurements made on JET during 1984 and 1985 with a ${ }^{3} \mathrm{He}$ ionization chamber spectrometer have been presented and the results discussed in terms of other available diagnostic data.

'Proceedings of the Second United Nations International Conference on the Peaceful Uses of Atomic Energy, Geneva, 1-13 September 1958 (IAEA, Vienna, 1958), Vol. 32.

${ }^{2}$ G. Lehner and F. Pohl, Z. Phys. 207, 83 (1967).

${ }^{3}$ H. Brysk, Plasma Phys. 15, 611 (1973); and loc. cit.

${ }^{4} J$. D. Strachan et al., Nature 279, 626 (1979).

W. A. Fisher, S. H. Chen, D. Gwinn, and R. R. Parker, Phys. Rev. A 28, 3121 (1983).

"O. N. Jarvis et al., Europhys. Conf. Abst. 9F, Part I, 223 (1985).

${ }^{7} \mathrm{C}$. Wharton, in Course in Plasma Diagnostics and Data Acquisition Systems, Varenna, 3-11 September 1975, p. 70.

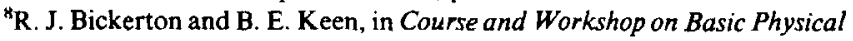
Processes of Toroidal Fusion Plasmas, Varenna, 26 August-3 September 1985 and JET-P( 85$) 24$.

${ }^{9} \mathrm{~J}$. D. Strachan et al., in Course and Workshop on Basic Physical Processes of 
Toroidal Fusion Plasma, Varenna, 26 August-3 September 1985.

${ }^{10} \mathrm{H}$. W. Hendel, in Diagnostics for Fusion Reactor Conditions, Varenna, 1982, EUR 8351 EN, 1982.

"O. N. Jarvis, in Diagnostics for Fusion Reactor Conditions, Varenna, 1982, EUR $8351 \mathrm{EN}, 1982$.

12J. Lorenzen, in Diagnostics for Fusion Reactor Conditions, Varenna, 1982, EUR 8351 EN, 1982.

${ }^{13}$ F. Hoenen, Nucl. Instrum. Methods Phys. Res. 223, 150 (1984).

${ }^{14} \mathrm{~K}$. Hübner et al., Europhys. Conf. Abst. 9F, Part I, 231 (1985).

${ }^{15}$ H. D. Kang and M. K. Chung, J. Kor. Phys. Soc. 8, 9 (1975); and in IAEA-R-1652-F September 1978.
${ }^{16} G$. Grosshoeg et al., Chalmers University of Technology, Sweden, reports CTH-RF-43, 1983 and CTH-RF-54, 1985.

${ }^{17} \mathrm{~J}$. Källne and T. Elevant, JET-P(85)03.

${ }^{18} \mathrm{~J}$. Scheffel, Nucl. Instrum. Methods Phys. Res. 224, 519 (1984).

${ }^{19} \mathrm{D}$. Slaughter, UCRL-82158, 1979.

${ }^{20}$ Jordan Valley, Emek Hayarden, Israel Spectrometer type FNS-1.

${ }^{21}$ W. Cash, Astrophys. J. 228, 939 (1979).

${ }^{22}$ V. Merlo, in Course and Workshop on Basic Physical Processes of Toroidal Fusion Plasma, Varenna, 26 August-3 September 1985.

${ }^{23}$ B. Denne et al., Europhys. Conf. Abst. 9F, Part I, 379 (1985).

${ }^{24} \mathrm{~S}$. Corti (private communication). 